\title{
Pratiques
}

Linguistique, littérature, didactique

141-142 | 2009

La synonymie

\section{Les synonymes de dans l'ensemble: de la généralité à la quantification totale, en passant par l'approximation}

Marie Lammert et Hélène Vassiliadou

\section{OpenEdition}

Journals

Édition électronique

URL : https://journals.openedition.org/pratiques/1343

DOI : $10.4000 /$ pratiques. 1343

ISSN : 2425-2042

Éditeur

Centre de recherche sur les médiations (CREM)

Édition imprimée

Date de publication : 15 juin 2009

Pagination : 179-194

Référence électronique

Marie Lammert et Hélène Vassiliadou, «Les synonymes de dans l'ensemble : de la généralité à la

quantification totale, en passant par l'approximation », Pratiques [En ligne], 141-142 | 2009, mis en ligne le 20 juin 2014, consulté le 10 mars 2023. URL : http://journals.openedition.org/pratiques/1343 ; DOI : https://doi.org/10.4000/pratiques. 1343 


\section{Les synonymes de dans l'ensemble: de la généralité à la quantification totale, en passant par l'approximation}

\section{Marie Lammert \& Hélène Vassiliadou}

Université de Strasbourg

EA 1339 LiLPa-Scolia

\section{Introduction}

Notre contribution à ce volume sur la synonymie se situe à un niveau descriptif et n'apporte de ce fait que peu d'eau au moulin théorique. Le but de cet article est en effet de confirmer, de spécifier ou d'infirmer les relations synonymiques établies par les dictionnaires entre l'adverbial dans l'ensemble et d'autres expressions généralement données comme équivalentes. Dans cette perspective, après avoir exposé la méthodologie suivie pour cette étude, nous procéderons à une brève description du fonctionnement syntactico-sémantique de dans l'ensemble qui nous servira de base dans sa confrontation avec ses dits « synonymes ». Ceux-ci touchent, selon les dictionnaires, des domaines quelque peu différents, à savoir la généralité (en général / généralement), 1'approximation (globalement / en gros / grosso modo), ou encore la quantification totale (au total / en bloc), autant d'aspects qui se révèlent éclairants sur le sémantisme de cette locution. Ces investigations auront pour conséquence de circonscrire plus avant non seulement le sens et les conditions d'emploi de dans l'ensemble, mais aussi ceux des autres adverbiaux.

\section{Méthodologie et remarques générales}

Il est bien connu en linguistique que la synonymie absolue est un fait isolé. D'autres articles dans ce volume (et ailleurs : $c f$. à titre d'exemple, Lyons, 1970, Lehmann \& Martin-Berthet, 1998, Le Français Moderne, 2007) traitent de ce problème d'un point de vue purement théorique. La synonymie est principalement considérée comme une relation de similarité ou de ressemblance sémantique entre deux ou plusieurs formes, impliquant des cas d'identité partielle (cf. Geerarts, 1994 : 4455, Haßler, 2005, Huyghes, à par.). Pour les locutions examinées ici, on peut constater qu'elles fonctionnent comme quasi-synonymes dans certains contextes, mais qu'elles ont aussi des emplois spécifiques. Ceci n'est pas très original en soi, puisque selon la théorie classique « les termes synonymes présentent tous 
une même idée principale, mais chacun d'eux y ajoute néanmoins quelques idées accessoires, qui diversifient la principale » (Girard, 1718 : xxvii ; cf. aussi Cruse, 2000 et Edmonds \& Hirst, $\left.2002^{(1)}\right)$. Il serait alors artificiel de vouloir montrer que les locutions adverbiales que nous examinons n'entretiennent pas de relations de synonymie stricte. Notre objectif se situe à un autre niveau : nous nous interrogeons sur leur degré de proximité sémantique et les causes de leur rapprochement, sur l'existence ou non d'une signification commune et sur les propriétés distinctives de chacune. Il s'agit donc d'étudier une gamme de locutions qui « se forge(nt) par constellation » (Rossari, 1996:56; $f f$. aussi Vassiliadou, $2008: 36$ ) et qui sont traitées comme synonymes dans les dictionnaires de référence. Le tableau 1 fait état des données collectées dans ces derniers :

\begin{tabular}{|l|l|l|l|}
\hline Dans l'ensemble & $\begin{array}{l}\text { Paraphrase } \\
\text { définitoire }\end{array}$ & $\begin{array}{l}\text { Définition } \\
\text { synonymique }\end{array}$ & $\begin{array}{l}\text { Renvois } \\
\text { et synonymes }\end{array}$ \\
\hline $\begin{array}{l}\text { Dictionnaire de l'Académie } \\
\text { (9e édition) }\end{array}$ & $\begin{array}{l}\text { d'une manière } \\
\text { générale }\end{array}$ & \\
\hline Larousse (2008) & en général & \\
\hline Nouveau Littré (2007) & de manière générale & $\begin{array}{l}\text { renvoi analogique : } \\
\text { globalement } \\
\text { (cf. au total, } \\
\text { en bloc, en gros) }\end{array}$ \\
\hline $\begin{array}{l}\text { Petit Robert électronique } \\
(\mathbf{2 0 0 1})^{(2)}\end{array}$ & $\begin{array}{l}\text { en considérant } \\
\text { plutôt l'ensemble } \\
\text { que les divers } \\
\text { composants }\end{array}$ & $\begin{array}{l}\text { en général, } \\
\text { d'une façon } \\
\text { générale }\end{array}$ & \\
\hline TLFi & & & $\begin{array}{l}\text { au total, } \\
\text { en gros }\end{array}$ \\
\hline $\begin{array}{l}\text { Dictionnaire des synonymes } \\
\text { du Crisco }\end{array}$ & & $\begin{array}{l}\text { au total, } \\
\text { en gros }\end{array}$ \\
\hline Atlas sémantique & & \\
\hline
\end{tabular}

Tableau 1 : Dans l'ensemble dans les dictionnaires

On note tout d'abord que, mis à part le $P R E$, aucune véritable paraphrase définitoire de dans l'ensemble n'est donnée : le definiens est la plupart du temps constitué par un autre adverbial (ce qui, comme on sait, conduit à une certaine circularité), cet adverbial relevant systématiquement de la généralité. Parmi les dictionnaires de langue, seul le $P R E$ renvoie à d'autres domaines, rejoignant ainsi les dictionnaires électroniques des synonymes.

Puis, il nous semble également intéressant, étant donné les définitions synonymiques qui en sont fournies par les dictionnaires, de faire un détour du côté de dans son ensemble / dans leur ensemble illustrés par (1):

(1) On dépose sur le dessus d'une petite armoire du linge porté par la mère d'un leader. On filme les réactions du groupe dans son ensemble. (F. Dolto, La Cause des enfants, 1985)

(1) «Usually words that are close in meaning are near-synonyms (or plesionyms) - almost synonyms, but not quite ; very similar, but not identical, in meaning : not fully intersubstitutable, but instead varying in their shades of denotation, connotation, implicature, emphasis or register. " (Edmonds \& Hirst 2002 : 107).

(2) Le Petit Robert rapproche également dans l'ensemble de en somme dans des éditions antérieures, et dans cette édition il fait aussi un renvoi à tout compte fait après un exemple. 
Une certaine confusion paraît régner entre dans l'ensemble et dans son ensemble, comme en témoigne le tableau 2 :

\begin{tabular}{|l|l|l|l|}
\hline $\begin{array}{l}\text { Dans son ensemble, } \\
\text { dans leur ensemble }\end{array}$ & $\begin{array}{l}\text { Paraphrase } \\
\text { définitoire }\end{array}$ & $\begin{array}{l}\text { Définition } \\
\text { synonymique }\end{array}$ & $\begin{array}{l}\text { Renvois } \\
\text { et synonymes }\end{array}$ \\
\hline $\begin{array}{l}\text { Dictionnairede l'Académie } \\
\text { (9e édition) }\end{array}$ & globalement & \\
\hline Larousse (2008) & $\begin{array}{l}\text { dans les grandes } \\
\text { lignes; entièrement }\end{array}$ & en gros, au total & \\
\hline Larousse (2001) & totalement & $\begin{array}{l}\text { 1. complètement, } \\
\text { intégralement, } \\
\text { totalement }\end{array}$ \\
\hline Nouveau Littré (2007) & & dans sa globalité \\
\hline (2001)
\end{tabular}

Tableau 2 : Dans son / leur ensemble dans les dictionnaires

Certains rapprochements sémantiques semblent en effet biaisés, dans leur conception de dans son ensemble, par le sémantisme de dans l'ensemble (cf. dans les grandes lignes vs complètement, intégralement, totalement). Le lien sémantique et formel entre dans son ensemble et dans l'ensemble est indéniable, mais ils se distinguent par deux points importants. D'une part, dans son ensemble, en raison du possessif, entretient une relation anaphorique stricte avec son antécédent et est rattaché, sémantiquement et syntaxiquement, à un nom, défini pour la plupart des cas, tandis que dans l'ensemble n'oblige plus, en tant que locution figée ${ }^{(3)}$, à nouer une telle relation. Il ne connaît pas, en d'autres termes, un tel lien avec un élément précis. D'autre part, dans son ensemble renvoie à la totalité, à l'intégralité du N auquel il se rattache et ne laisse pas place aux exceptions, contrairement à dans l'ensemble qui, comme nous le verrons ci-après, n'atteint jamais la totalité.

Ces différences nous poussent à écarter dans son ensemble de cette étude, pour nous concentrer sur les adverbiaux donnés comme équivalents de dans l'ensemble. Par les différents parallèles effectués, nous tenterons de mettre en lumière les spécificités syntactico-sémantiques ${ }^{(4)}$ de dans l'ensemble et d'examiner en quoi réside son originalité par rapport aux autres. Nous nous plaçons ainsi dans la perspective de Cruse (2000 : 156) et Kleiber (dans ce volume) selon laquelle les synonymes sont des mots dont les similarités sémantiques sont plus saillantes que leurs

(3) Sur ce point, $c f$. Lammert et Vassiliadou (à par.).

(4) Blumenthal $(2002: 116)$ utilise à ce propos le terme de profil combinatoire défini comme « la structure schématique du voisinage syntaxique et sémantique d'un mot telle qu'elle se manifeste dans un vaste corpus ». Le corpus utilisé pour notre étude est, d'une part, Frantext et, d'autre part, les corpus oraux de Lyon (Clapi) et d'Orléans (Elicop). 
différences. Nous allons mettre au jour les points communs particuliers qui font qu'on peut rapprocher dans l'ensemble de tous ces adverbiaux, le rapprochement ne se faisant pas sur les mêmes bases pour tous les items. Ceci nous permettra également d'apporter des éléments de réponse quant aux types de différences qui bloquent ou non une intuition de similarité entre eux ( $c f$. Cruse, 2000 : 156). Dans cette optique, notre analyse devra non seulement prendre en compte le sémantisme des unités, mais également leurs propriétés morphosyntaxiques ( $c f$. aussi Fuchs, 2007 : 97). Cela est d'autant plus important que les adverbiaux ne font pas partie des catégories prototypiques touchées par la synonymie, telles que les noms, les adjectifs ou les verbes.

\section{Dans l'ensemble et ses équivalents}

Dans l'ensemble peut être considéré comme un marqueur d'atténuation au sens où il modalise la valeur de vérité d'un énoncé, l'utilisation de ce marqueur supposant simultanément un jugement et un calcul. Par son sémantisme quantificationnel (Lammert et Vassiliadou, à par.), il fait intervenir un processus qui lui est propre, processus que 1'on peut exemplifier avec l'énoncé suivant :

(2) Dans l'ensemble, les vacances étaient agréables.

Dans (2), avant de pouvoir énoncer dans l'ensemble, il est supposé qu'on a procédé à l'estimation des critères préexistants qui évaluent des vacances agréables, tels que le temps, l'ambiance, la qualité de la nourriture, les activités culturelles et autres, etc. Cette énumération de critères, mettant en jeu des traits essentiels et des traits mineurs, est une opération mentale latente qui amène à une conclusion, celle-ci ne s'établissant qu' après avoir considéré l'ensemble des traits. Cette évaluation permet de faire abstraction de détails qui ne répondraient pas positivement aux critères requis : avec dans l'ensemble, on sait donc que certains des critères ne sont pas remplis, mais on considère que leur absence n'est pas décisive pour que des vacances soient agréables, ce qui fait qu'ils peuvent être qualifiés de détails. Le rapport entre le sujet et le prédicat admet ainsi une modalisation « quantificationnelle », celle-ci se traduisant par l'existence d'exceptions, à savoir les « détails » qui ne vérifient pas les traits essentiels de la prédication et qui peuvent être soit explicites, soit implicites. Le nombre et la nature des détails ne sont alors pas connus, mais laissés à l'approximation.

Les détails dont il est question doivent cependant rester des détails ou, pour le dire autrement, la quantité de détails à partir de laquelle s'établit dans l'ensemble doit être faible. Il existe aussi une hiérarchisation des traits pris en compte. Cette expression construit ainsi un ordre de grandeur ${ }^{(5)}$ correspondant aux détails qui peuvent être pris en considération sans que la valeur de vérité de l'énoncé soit mise à mal. L'énoncé (2) analysé ci-dessus, par exemple, suppose d'un côté que les éléments qui font que des vacances ne sont pas agréables sont en nombre inférieur par rapport à ceux qui font qu'elles le sont, et d'un autre côté que les conditions essentielles pour que des vacances soient agréables sont remplies. Nous n'avons cependant pas affaire à une quantification stricto sensu, car on ne compte pas le

(5) L'effet d'approximation provoqué par dans l'ensemble est renforcé par la préposition dans qui, dans des quantificateurs prépositionnels (Il aurait reçu dans les trente propositions) construit, selon Mélis (2003 : 10-11), un intervalle, un ordre de grandeur, donc une quantification approximative. 
faible nombre qui ne vérifie pas le prédicat, mais on oppose niveau d'ensemble et niveau de détail. Dans ce sens, l'approximation coïncide avec l'imprécision.

L'opération de quantification qui s'établit avec dans l'ensemble nécessite ainsi la prise en compte à la fois du rapport entre le sujet et le prédicat et des connaissances stéréotypiques qui y sont généralement associées, cette quantification étant laissée à l'approximation. Ce marqueur prend donc en compte aussi bien un niveau général ou niveau d'ensemble, qu'un niveau faisant intervenir les éléments qui font cet ensemble ou niveau de détail. Il y a ainsi dans son sémantisme l'idée d'une quantification par approximation, mais le résultat de cette opération conduit à l'atténuation.

\subsection{Généralement-en général : faible nombre, généricité et habitualité}

La locution qui est le plus souvent citée comme synonyme de dans l'ensemble est en géneral (ou encore généralement, de façon / manière générale, en règle générale). Cette expression rejoint en effet le domaine de l'approximation par le biais de la même paraphrase que l'on peut appliquer à dans l'ensemble, à savoir que « pour pouvoir dire en général, il faut laisser place à quelques possibles exceptions » (Anscombre, $1990: 233 ; c f$. aussi Molinier, 2005). De prime abord, dans l'ensemble partage avec généralement la lecture «il est généralement vrai» (proche de la variante «d'une façon générale »), mais pas dans tous les cas. De fait, si nous pouvons remplacer dans l'ensemble par généralement dans 1'exemple (3), l'effet de sens produit n'est pas le même :

(3) Les scandinaves sont dans l'ensemble / généralement blonds.

Ici généralement porte plutôt sur le faible nombre que sur le détail et reçoit en plus une lecture générique ${ }^{(6)}$ :

(4) Les scandinaves sont habituellement blonds.

Cette lecture est impossible avec dans l'ensemble qui accentue surtout l'existence d'un ensemble. En outre, il a bien été montré dans la littérature ( $c f$. surtout Lewis, 1975 et Kleiber, 1983 et 1987) que généralement porte sur les occasions (ou cas). Nous pouvons alors appliquer la paraphrase « dans la plupart des cas où p est vrai ». Nous avons affaire, selon la terminologie de Lewis (1975:8), à un « quantificateur non sélectif» qui « li[e] sans discrimination toutes les variables qui se trouvent dans [son] champ » (Kleiber, 1983 : 189). En revanche, la lecture habituelle est bloquée avec dans l'ensemble qui, pour (3), acceptera plutôt la paraphrase « la plupart des scandinaves ». De même, en (5):

(5) Dans l'ensemble/ Généralement Paul est sympathique.

(6) Cette caractéristique n'est évidemment pas nouvelle. Elle est mise en avant dans Kleiber (1987) ainsi que dans l'étiquette d' «adverbe d'habitude » chez Molinier et Levrier (2000) et Molinier (2005). Chez Nøjgaard (1993: 415, § 567), nous trouvons l'appellation d'« itératif normatif ", à savoir d'adverbe qui a la capacité de "rapporter un membre de phrase à un 'paradigme', c-à-d à une échelle de fréquence ». A l'instar de Molinier (2005:91), notons également que même si généralement / en général font partie de la classe des adverbes d'habitude, ils sont « les plus abstraits » au sens où ils « attribuent seulement un ordre de grandeur à la régularité d'un phénomène, la régularité maximale », mais ils ne rapportent pas celle-ci à «l'ordre familier des choses » comme ordinairement par exemple ou encore à la norme, à la tradition, etc. (cf. normalement, traditionnellement, etc.). De cette manière, ils peuvent rester proches de dans l'ensemble. 
dans l'ensemble fait intervenir en plus un niveau de jugement qui amène le locuteur à «calculer » les traits de la personnalité de Paul qui font qu'il a la propriété d'être sympathique. Il est certes également possible pour généralement de porter tantôt sur les individus tantôt sur le temps, selon les variables contenues dans $\mathrm{p}$, mais quand on a un SN sujet spécifique, cet adverbe « ne peut lier que la variable temporelle » et la phrase n'est susceptible que d'une interprétation $\sim \forall$ t ( $c f$. Kleiber, 1983 : 191). En d'autres termes, nous aurons une quantification temporelle (aspect habituel quasi-universel) qui correspond à la lecture « dans la plupart des cas où Paul est sympathique » ou, pour reprendre l'analyse de Kleiber (1983), « Paul est [sympathique] à t». Ainsi, être sympathique associé à dans l'ensemble est conçu comme une propriété, tandis qu'avec généralement il reçoit une lecture d'état occasionnel. En somme, même si le résultat est quasiment le même avec dans l'ensemble et généralement, le processus via lequel les deux adverbiaux l'atteignent est sensiblement différent. Ce qui nous amène à la distinction suivante : on voit encore une fois que dans l'ensemble, même s'il appartient aux adverbes de phrase ${ }^{(7)}$, est sémantiquement plus proche du $\mathrm{N}$ auquel il se rapporte, tandis que généralement est un quantificateur pur qui exige des cas :

(6) Le dernier livre de Kundera est dans l'ensemble/* généralement /* en général intéressant.

(7) Les livres de Kundera sont dans l'ensemble / généralement /en général intéressants.

Dans (6), le livre en question est pris comme une unité avec des parties ce qui peut correspondre à « le livre pris dans son ensemble».

Ainsi, dans l'ensemble est plus proche des quantificateurs quasi-universels non génériques (tels que la plupart de, le plus souvent), qui, à 1'opposé de généralement, " n'ont pas la capacité d'entraîner par eux-mêmes la généricité de la phrase » (Kleiber, $1988: 5$ ). Cette propriété de dans l'ensemble fait que, même s'il donne à penser qu'il y a des occurrences qui ne vérifient pas le prédicat ( $c f$. niveau de détail), il est paradoxalement compatible avec des marqueurs d'exception tels que sauf, à l'exception de, hormis, etc., ce qui n'est pas le cas de généralement:

(8) Dans l'ensemble, hormis quelques types étranges (tel un pseudo-machairodus récemment trouvé fossile en Patagonie) ce biote marsupial a disparu sans laisser de traces. (P. Teilhard de Chardin, Le Phénomène humain, 1955)

(9) Dans l'ensemble il apparaît que, à l'exception de quelques formes franchement endogées, les limivores ingèrent simultanément le film et une partie plus ou moins importante du sédiment sous-jacent. (J.-M. Pérès, La Vie dans l'océan, 1966)

Sur ce point, dans l'ensemble partage sa compatibilité avec les exceptions avec les phrases génériques comme (10):

(10) Les Alsaciens sont des buveurs de bière, sauf / hormis les strasbourgeois. (Kleiber, $1988: 11)$

Dans l'ensemble rejoint ainsi d'une certaine manière les quantificateurs quasiuniversels, mais il reste avant tout un modalisateur qui a surtout besoin, en combinaison avec le prédicat, de s'appliquer à une situation globale et d'affecter ou de caractériser l'ensemble des occurrences concernées par le contexte, tout en faisant abstraction de certains traits.

(7) $C f$. Lammert et Vassiliadou (à par.) pour une analyse syntaxique détaillée. 


\subsection{En gros et grosso modo : résumé approximatif}

En gros et grosso modo ${ }^{(8)}$ représentent le deuxième type d'équivalence donné par les dictionnaires à propos de dans l'ensemble. Nous pouvons d'ores et déjà dire que ce rapprochement est justifié par le sémantisme de ces deux locutions qui signale une approximation via la prise en compte d'un niveau de détail :

(11) Celui qui aime la littérature n'aime ni l'argent, ni les tableaux, ni les bibelots, ni le reste. Au fond, Balzac n'aimait pas la littérature. Balzac est vrai en gros, il ne l'est pas en détail. Des nuages gris, veloutés, et comme illuminés de feux intérieurs. (J. Renard, Journal, 1895)

Toutefois, le processus par lequel en gros fait référence indirectement aux détails n'est pas le même : dans l'ensemble indique une sorte d'addition de traits qu'on pourrait qualifier d'importante au sens où on a un cumul de détails au terme duquel on peut dire que l'essentiel l'emporte, ou, pour le dire autrement, l'ensemble l'emporte sur les détails. Avant de pouvoir énoncer dans l'ensemble, il est supposé qu'on a procédé à une estimation de critères qui évaluent $X$. Cela nous amène ainsi à postuler l'existence d'éléments mineurs, et d'un ensemble qui peut être préconstruit et préexistant ${ }^{(9)}$ et par conséquent être extérieur à la prédication. Mais on a toujours affaire à un ordre argumentatif croissant (quand il y a des [+] et des [-], il y a quand même plus de [+] que de [-]). Dans l'ensemble tire ainsi vers le positif. En gros, quand il peut se mettre à la place de dans l'ensemble, indique que le calcul de traits n'est pas aussi essentiel, il se fait de manière grossière. Les traits en question ne sont pas hiérarchisés, ou mieux, ne sont pas affinés. En gros comporte l'idée de simplification, d'où les diverses occurrences dans notre corpus du type simplifions les choses et disons qu'en gros...

Ainsi, si on substitue en gros à dans l'ensemble dans un exemple comme (12), la différence est assez nette : avec dans l'ensemble, on laisse entendre qu'il y a quand même plus de choses justes dans l'intuition de Gougenheim qu'avec en gros :

(12) L'intuition de Gougenheim est dans l'ensemble / en gros ${ }^{(10)}$ juste.

De ce point de vue, dans l'ensemble fonctionne à la manière de tout compte fait au sens où il déclenche un travail «d'élaboration conceptuelle » plus important de la part du locuteur ( $c f$. Charolles, 1984 : 97, Rossari, 1993 : 154), tandis qu'en gros laisse entendre qu'on évacue les détails en effectuant en quelque sorte une opération résomptive, à la manière de $b r e f(c f$. Schnedecker, 1992). Nous pouvons sentir ces différences d'effets de sens dans les exemples suivants provenant de forums sur la toile :

(13) Bordeaux a eu de bons résultats dans l'ensemble / en gros.

(14) C'est un bon caméscope dans l'ensemble / en gros.

(15) Wokfu (jeu vidéo) c'est cool dans l'ensemble / en gros.

(8) En gros et grosso modo semblent s'employer dans tous les contextes exactement de la même manière, en tant que synonymes absolus. Ceci est probablement dû au fait qu'on a affaire à une locution latine et son correspondant français. De ce fait, les remarques que nous ferons à propos de en gros vaudront également pour grosso modo.

(9) Nous remarquons à cet effet que les phrases du type «c'est ça en gros» ou « la réponse, c'est en gros que... » sont plus naturelles que «c'est ça dans l'ensemble» justement à cause de la difficulté de voir un ensemble quelconque.

(10) Lorsque nous mettons en parallèle deux ou plusieurs adverbiaux, c'est celui qui figure en première position qui est présent dans l'énoncé attesté. 
En gros et dans l'ensemble, dans ce type de contextes partagent l'effet de clôture (également commun à au total, $c f$. § 2.4.) qui « va de pair avec la présupposition d'une accumulation ou d'une succession d'événements, de preuves, de réflexions, qui précèdent cet acte de clôture » (Rossari, 1993 : 152). Toutefois, dans l'ensemble reste proche du GN de la phrase et agit comme un modalisateur d'atténuation mettant en perspective que $\mathrm{X}$ est considéré dans sa globalité, dans ses différents aspects et non d'une manière grossière.

De plus, en gros s'interprète mieux si on lui attribue un contexte plus large sur lequel il peut enchaîner avant d'énoncer, comme dans (14), que Xest un bon caméscope. Il fonctionne davantage comme un connecteur de reformulation qui introduit un résumé (fonction récapitulative). Il présente ainsi un caractère métalinguistique lui conférant non seulement cette capacité rétroactive, mais aussi celle de signaler un commentaire sur la forme de l'énoncé ( $c f$. dire en gros) ou « sur le choix des formes linguistiques » (Guimier, 1996 : 124) :

(16) Car le passé défini a disparu de la langue parlée, mais la langue littéraire continue à l'imposer ; cette acquisition se fait par la lecture et l'écriture c'est-à-dire, en gros, d'adultes à adultes. (C. Bally, Le Langage et la vie, 1913)

(17) A : de tous les temps euh la géographie euh toutes sortes de choses tout tout est intéressant dans le dictionnaire.

$\mathrm{B}$ : vous le regardez à peu près combien de fois dans le mois par exemple approximativement?

A : je passe une semaine sans y mettre le nez et puis quelquefois je je l'ouvrirai deux trois fois par euh par semaine ça dépend.

B : oui . alors en gros ça doit faire à peu près une fois je sais pas une fois par semaine? (Elicop)

(18) Après la poterie, l'art floral, la graphologie, la reliure, c'est le tour de la photographie. Qu'elle discute, et parfois critique, la façon dont il gère ses biens et ceux de sa mère. Qu'elle renonce de plus en plus souvent aux politesses de façade. En gros, il remarque qu'elle ne le remarque plus. Que c'est lui qui est devenu pour elle un objet transparent, une quantité négligeable. (F. Dorin, Les Vendanges tardives, 1997)

(19) EMI : c'est-à-dire qu'en fait si des gens ont besoin de : : ne sont pas satisfaits de leur vie ailleurs et que : : en fait tu leur dis en gros restez dans vot' merde moi euh MAR : non justement c'est pas ça alors là t'as pas tout entendu (Clapi)

Dans l'ensemble, au contraire, ne peut pas apparaître dans ce type d'exemples, car il n'est pas un élément conjonctif. On pourrait supposer, à l'instar de Rossari (1994 : 64), qu'il « n'a pas subi au même degré le processus de désémantisation qui conduit d'une fonction originelle d'opérateur à une fonction dérivée de connecteur ». Cette locution apporte surtout une modalisation ${ }^{(11)}$ à l'ensemble de l'énoncé dans lequel elle intervient. Nous pouvons d'ailleurs, comme nous le verrons par la suite, lui substituer systématiquement l'adverbe globalement, fait qui appuie sa fonction d'opérateur modal, apportant à l'énoncé une atténuation plutôt qu'une approximation. A l'inverse, en gros se comporte davantage comme un « approximateur», ce que montre aussi l'un des tests classiques, à savoir la combinaison possible avec tous les ${ }^{(12)}$ ( $c f$. Jayez et Tovena, $2008: 6$ ).

(11) Dans l'ensemble joue le rôle d'enclosure ou hedge, comme nous l'avons montré ailleurs.

(12) Dans l'ensemble est incompatible avec les quantificateurs universels (cf. Vassiliadou \& Lammert, 2008). 


\subsection{Globalement : de la complétude à la «globalité »}

L'examen des occurrences de globalement dans notre corpus fait ressortir différents emplois de cet adverbe. Celles-ci ont en commun de prendre en compte un ensemble (qui peut être, selon le contexte, relié à un ou des $\mathrm{N}$ particuliers ou être constitué par un ensemble de traits procédant du rapport entre le sujet et le prédicat), mais cet ensemble va être considéré et atteint différemment selon les acceptions de globalement. La constitution de cet ensemble transparaît dans des énoncés du type de (20) et (21), dans lesquels une dénomination est attribuée à l'ensemble des attitudes et des sentiments dont il est question :

(20) À partir de là, mais à partir de là seulement, on peut voir apparaître chez l'ouvrier parcellaire, à condition, bien entendu, qu'il ait été sélectionné et qu'il soit psychophysiologiquement bien adapté à ces tâches, des attitudes plus complexes qui se différencient de la simple conscience professionnelle et que nous désignons globalement par : satisfaction professionnelle. (G. Friedmann, Où va le travail humain?, 1950)

(21) Les actes de haute tension se signalent chez le normal par des sentiments de calme, de joie, d'intérêt, de confiance, d'indépendance, de triomphe, que Janet groupe sous le nom de «sentiments d'élation», et qui constituent les divers modes de ce que l'on appelle globalement l'humeur optimiste. (E. Mounier, Traité du caractère, 1946)

On peut en effet mettre en évidence différents niveaux d'implication de l'ensemble qui correspondent aux acceptions de globalement et qui sont en rapport avec sa portée syntaxique :

1) l'ensemble est affecté " entièrement », " en bloc », "dans son ensemble » (cf. 1'étiquette d'adverbe de complétude donnée par Molinier et Levrier, 2000 : 209-214); c'est un adverbe endophrastique :

(22) L'administration paie un franc vingt-cinq par journée d'homme avec charge, et soixante-quinze centimes l'homme non chargé ; mais souvent, la somme est remiseglobalement au chef, de sorte qu'il arrive que les intéressés ne touchent rien. (A. Gide, Voyage au Congo, 1927)

2) l'ensemble est concerné entièrement, mais c'est le jugement qui est effectué selon une "vue d'ensemble ", c'est-à-dire sans prendre en compte le niveau de détail, en laissant place à l'imprécision, en émettant un jugement « de manière globale », et jouant le rôle d'un adverbe endophrastique :

(23) Khrouchtchev a globalement estimé les pertes humaines de cette période à 10 millions. Mais la réalité dépasse sans aucun doute cette estimation. (H. Carrère d'Encausse, L'Empire éclaté, 1978)

3) l'ensemble n'est pas touché entièrement, et la portion d'ensemble qui n'est pas touchée est laissée à l'approximation, le jugement s'effectuant selon les mêmes modalités que dans le cas précédent ${ }^{(13)}$; il porte alors sur la phrase :

(24) Il n'y a aucune raison de supposer qu'après un crime aussi atroce que le meurtre d'un être parfait l'humanité ait dû devenir meilleure ; et en fait, globalement, elle ne semble pas être devenue meilleure. (S. Weil, Euvres, 1929-1943)

(13) Le DHLF note que globalement est attesté en 1840 avec le sens de « en bloc » et qu'il signifie aussi, à partir du $20^{\mathrm{e}}$ siècle, "d'une manière générale ". 
Précisons qu'il est parfois difficile de distinguer entre ces acceptions, et cela malgré les différences sémantiques qu'elles peuvent induire. L'occurrence de globalement dans l'exemple (25) pourrait être paraphrasée soit par « de manière globale », soit par « dans l'ensemble », et celle de (26) par « en bloc » ou « dans l'ensemble », produisant ainsi des effets de sens différents :

(25) Que ce soit dans la sphère de la vie privée ou dans celle de la vie publique, les événements dont nous avons été témoins ont globalement signifié un élargissement de la logique individualiste dénommée alors, non sans clin d'œil ironique, postmoderne. (G. Lipovetsky, L'Ėre du vide, 1983)

(26) Sans doute, le Parti communiste de l'U.R.S.S. est-il très prudent lorsqu'il en vient à préciser les crimes staliniens. S'il admet globalement la destruction des élites nationales, il s'efforce de ne pas ranimer le souvenir personnalisé de dirigeants nationaux, [...] (H. Carrère d'Encausse, L'Empire éclaté, 1978)

On peut mettre en évidence ces différences sémantiques par le biais des paraphrases déjà indiquées, mais aussi à travers les oppositions qu'elles vont induire. L'acception 1 de "complétude » s'oppose ainsi à un ensemble affecté partiellement, ceci pouvant être mis en évidence par un contexte du type :

(27) La somme est remise globalement au chef, et non pas partiellement / * et non pas entièrement.

(28) [...] les délégations doivent rester partielles, une autorité ne pouvant déléguer globalement toutes ses compétences [...] (G. Belorgey, Le Gouvernement et l'administration de la France, 1967)

(29) Mais tandis que Dieu aliénait globalement la subjectivité, la bourgeoisie l'émiette dans un ensemble d'aliénations partielles. En un sens, la subjectivité, qui n'était rien, devient quelque chose, [...] (R. Vaneigem, Traité de savoir-vivre à l'usage des jeunes générations, 1972)

Les acceptions 2 et 3 -qui impliquent l'imprécision - font intervenir un niveau d'ensemble, un niveau global qui prend en compte l'intégralité de l'ensemble, mais sans entrer dans les détails. Ce niveau d'ensemble peut alors être opposé à un niveau de détail qui transparaît dans les énoncés suivants (pour ce qui est de 1'acception 2):

(30) 9 juillet $1920:-$ peut-être la question de savoir ce que je vaux n'est-elle légitime qu'à condition d'être posée en détail et non globalement - et le métaphysicien se paie-t-il de mots en la soulevant en dehors des sphères particulières où elle conserve un sens. (G. Marcel, Journal métaphysique, 1923)

(31) elle marque un déficit; elle traduit une infirmité de notre perception, condamnée à détailler le film image par image au lieu de le saisir globalement. (H. Bergson, $L a$ Pensée et le Mouvant, 1934)

(32) C'est encore Toubin qui raconte (tout cela se présente en moi non pas textuellement, avec des mots et en détail, mais globalement, en une sorte de totalité immédiate, aussi précise et plus rapide qu'une citation)...(C. Mauriac, La Marquise sortit à cinq heures, 1961)

Par ailleurs, la vue globale, générale est explicitement mise au jour dans les énoncés suivants :

(33) $[\ldots]$ j'apercevais l'adulte qu'elle serait, qu'elle était déjà pour quelques-uns, comme si grâce à mon éloignement j'avais acquis le pouvoir de la découvrir globalement, en vue aérienne. (B. et F. Groult, Il était deux fois, 1968) 
(34) L'observation aérienne s'étend aux domaines les plus variés : relevés topographiques, associations animales et végétales dans leurs rapports avec les activités humaines, phénomènes de foule, documents de psychologie collective. Elle donne une vue d'ensemble qui permet de saisir globalement l'activité d'un groupe donné. La photographie aérienne révèle des vestiges archéologiques invisibles en observation terrestre : fonds de cabanes, palafittes, [...] (M. Daumas (dir.), Histoire de la science, 1957)

En dehors de la prise en compte d'un ensemble qui leur est commune, les différentes acceptions de globalement présentent une correspondance deux à deux :

- les acceptions 1 et 2 ont en commun la complétude,

- les acceptions 2 et 3 partagent un processus d'évaluation particulier fondé sur la prise en compte d'un niveau d'ensemble qui fait abstraction des détails, laissant place à l'imprécision.

La complétude commune aux acceptions 1 et 2 se révèle dans les expressions « pris globalement » ou «considéré globalement » présentes dans les énoncés suivants :

(35) [...] la nécessité de choisir est encore marquée par le conflit des valeurs vitales prises globalement avec l'ordre également global des valeurs sociales ; (P. Ricœur, Philosophie de la volonté, 1949)

(36) Un office de placement a deux sections : l'une, la section A où règne une forte compétition, et l'autre, $B$, où elle est très faible. Considérée globalement, la section $B$ produit plus que la section A [...] (G. Gurvitch, Traité de sociologie, 1967)

Quant au processus d'évaluation des acceptions 2 et 3, il est également présent dans le sémantisme de dans l'ensemble. Ainsi, le rapprochement de dans l'ensemble avec globalement ne peut se faire qu'avec l'acception 3, lorsqu'il est adverbe de phrase et que son sémantisme s'éloigne de la complétude, répondant alors aux propriétés sémantiques de dans l'ensemble. Ce mode de fonctionnement commun apparaît dans les énoncés mettant en évidence l'opposition entre niveau d'ensemble et niveau de détail, comme nous l'avons vu concernant globalement dans les exemples (30) à (32), mais aussi dans les énoncés où dans l'ensemble est mis en parallèle avec le détail. Dans les deux cas, dans l'ensemble et globalement sont interchangeables:

(37) Elle est émouvante, vénérable dans l'ensemble / globalement, en tant qu'animée par les dieux, et j'en fait mon profit, mon plaisir dans les détails. (M. Barrès, Mes Cahiers, 1918)

(38) [...] comme le plus intime, le plus sûr de ses confidents nous l'affirme dans une page étonnante, qui appelle sans doute quelques atténuations de détail, mais qui, dans l'ensemble / globalement, me paraît inattaquable. (H. Bremond, Histoire littéraire du sentiment religieux, 1921)

L'interchangeabilité ne nécessite cependant pas ce contexte particulier, puisqu'elle est possible dans toutes les acceptions 3 de globalement, et dans toutes les occurrences de dans l'ensemble:

(39) [...] leurs bombardiers Glenn-Martin venaient se poser sur le terrain d'aviation et le général Têtu les donnait comme l'avant-garde de ce qui allait bientôt suivre. Globalement / Dans l'ensemble, le résultat était, cependant, favorable. (C. de Gaulle, Mémoires de guerre, 1954) 
(40) $\mathrm{La} \mathrm{V}^{\mathrm{e}}$ République a bénéficié de cet acquis ; de plus, la situation du monde occidental était globalement / dans l'ensemble assez bonne (avec naturellement des fluctuations) et nous en avons profité. (P. Mendès-France, Euvres complètes, 1990)

(41) Oui, ils ont, dans l'ensemble / globalement, plus de cœur, plus d'humanité que les autres ; vertus de vaincus... ce qui est bien certain, c'est que je n'ai qu'un dégoût haineux pour la bourgeoisie dont je sors. (A. Malraux, Les Conquérants, 1928)

(42) [...] de toutes façons, je n'essayais pas de me battre pour être première. Ça ne m'intéressait pas. Pourquoi être première ? Ce que les gens pensaient de moi m'était dans l'ensemble / globalement bien égal. (C. Rochefort, Les Petits enfants du siècle, 1961)

Globalement fonctionne alors, à la manière de dans l'ensemble, comme un modalisateur d'atténuation. Mais, d'un point de vue discursif, dans l'ensemble, en tant que locution adverbiale composée de dans + l'ensemble, permet, de mettre en opposition les séquences " dans le(s) détail(s)»/ " dans l'ensemble », comme on peut le voir dans l'exemple (37). De son côté, l'adverbe globalement présente la possibilité d'être suivi du participe parlant, marquant ainsi explicitement « le caractère externe à la phrase » (Guimier, 1996:12) de cet emploi, de même que la paraphrase par « une construction incidente, parenthétique, qui marque un commentaire de l'énonciateur » (Guimier, $1996: 12$ ) du type d'un point de vue global :

(43) Dans le moyen terme - entre 1950 et 1975 - et globalement parlant, il s'agit de fournir une nourriture adéquate à 1330 millions d'êtres humains supplémentaires. (F. Perroux (dir.), L'Univers économique et social, 1960)

(44) Il y a des taches énormes de pauvreté, de misère, mais, globalement parlant, le pays est plus riche qu'il ne l'était il y a une génération, c'est tout à fait vrai... (P. MendèsFrance, Euvres complètes, 1990)

\subsection{Au total: de la quantification à la reformulation}

Le quatrième type de parallèle dont dans l'ensemble est à l'origine prend en compte une expression liée à la quantification totale qu'est au total. Le parallèle ainsi fait ne concerne cependant que l'emploi noté comme figuré de au total ${ }^{(14)}$, celui-ci étant décrit par Schnedecker (2008) comme un marqueur de reformulation introduisant un bilan « auquel aboutit le locuteur après avoir passé en revue la totalité des arguments, qu'ils plaident en faveur ou contre la conclusion visée ». Il est illustré par les exemples suivants :

(45) Ils ont été beaucoup critiqués pour leurs défauts, ou pour leurs soi-disant défauts, mais, au total, depuis le jour où la Convention Nationale a décrété la naissance de l'École polytechnique, jusqu'au jour où Joffre, Foch, Fayolle défilèrent sous l'Arc de Triomphe, l'École a bien servi la France. (C. de Gaulle, Discours et messages, 1970, cité par Schnedecker, 2008 : 135-136)

(46) La ligne sèche, austère et tendue - au total admirable - de l'épure dramatique de Molière est ici détrempée de bout en bout (le merveilleux dernier acte mis à part) par le climat sentimental du dix-huitième siècle finissant. (J. Gracq, Carnets $d u$ grand chemin, 1992)

(14) Il existe en effet deux emplois de au total mis en évidence par Schnedecker (2008), l'un comme quantificateur adverbial (elle a bu trois whiskies au total), l'autre comme marqueur de reformulation introduisant un bilan. C'est le second que nous prendrons en compte ici. 
Il est en effet possible, dans ce type d'énoncés, d'utiliser dans l'ensemble, le changement d'adverbial introduisant des différences sémantiques notables :

(47) Ils ont été beaucoup critiqués pour leurs défauts, ou pour leurs soi-disant défauts, mais, dans l'ensemble, depuis le jour où la Convention Nationale a décrété la naissance de l'École polytechnique, jusqu'au jour où Joffre, Foch, Fayolle défilèrent sous l'Arc de Triomphe, l'École a bien servi la France.

(48) La ligne sèche, austère et tendue - dans l'ensemble admirable - de l'épure dramatique de Molière est ici détrempée de bout en bout (le merveilleux dernier acte mis à part) par le climat sentimental du dix-huitième siècle finissant.

Au total et dans l'ensemble ont en commun leur caractère conclusif : ils évaluent tous les deux un certain nombre d'éléments avant de pouvoir être énoncés et d'établir, par leur utilisation, une sorte de bilan. Le comparatif ainsi fait permet d'appuyer l'analyse sémantique de dans l'ensemble comme lieu d'un processus d'évaluation. Ce qui est pris en compte diffère cependant largement dans les deux cas. Au total est un connecteur qui présuppose un matériau discursif antérieur, explicite ou implicite, tandis que dans l'ensemble évalue le rapport existant entre le sujet et le prédicat (et les connaissances stéréotypiques qui y sont associées) de l'énoncé dans lequel il est inséré. Cette différence fait que au total ne peut pas être utilisé dans tous les contextes où dans l'ensemble est employé, les conditions d'établissement de l'évaluation d'au total nécessitant un contexte plus large :

(49) Les peuples primitifs ont dans l'ensemble / ? au total atteint le même degré de développement que les hautes civilisations de l'antiquité qui se contentaient d'un simple vêtement. (R.-H. Lowie, Anthropologie culturelle, 1936)

(50) Beaucoup d'entre eux y adhèrent parce qu'elle répond, dans l'ensemble / ? au total, à d'anciennes revendications. D'autres l'acceptent comme une concession accordée à la paix sociale. (C. de Gaulle, Mémoires de guerre, 1959)

Par ailleurs, le bilan introduit ne se fonde pas sur le même mode opératoire pour ce qui concerne au total et dans l'ensemble (ainsi que globalement). Sur le modèle de l'analyse proposée pour en somme (Steuckardt, $2005: 281$ ), on peut dire que au total « introduit le résultat de la pesée [de] différents éléments [...] sans pour autant dégager à proprement parler un [«total»], mais plutôt un reste, un bilan ». Dans l'ensemble, en revanche, n'indique ni une opération de sommation au sens strict, ni une considération individuelle des unités faisant partie de l'ensemble, mais un traitement global de ce qui est considéré.

\subsection{En bloc: la question de la complétude}

Certains dictionnaires proposent un parallèle entre dans l'ensemble et en bloc qui nous semble devoir être modulé. Sémantiquement, en bloc peut être considéré comme un marqueur de totalité et non comme un marqueur d'approximation. Dans les énoncés (51) et (52),

(51) Elle condamne en bloc sa classe sociale, mais aussi les autres. (P. Sollers, Le Secret, 1993)

(52) Au fond, je suis sûre d'une chose aujourd'hui : le malheur se déguste goutte à goutte ou en bloc mais il faut avaler sa ration. (B. et F. Groult, Il était deux fois, 1968)

une substitution par dans l'ensemble, lorsqu'elle est possible, induit une interpré- 
tation sémantique tout autre, puisque la totalité n'est dans ce cas plus atteinte. Notons également que, syntaxiquement, en bloc est un adverbe endophrastique qui ne peut avoir d'emplois exophrastiques, différant ainsi de dans l'ensemble. Ces deux locutions sont donc trop éloignées pour pouvoir être considérées comme des synonymes ou pour qu'un renvoi puisse être fait de l'une à l'autre.

On peut cependant expliquer le rapprochement ainsi effectué par deux phénomènes. Tout d'abord, à travers le lien sémantique fait entre les items bloc et ensemble. Ensuite, via les renvois synonymiques communs qu' on leur attribue : dans l'ensemble est rapproché de globalement, qui lui-même est mis en relation avec en bloc et inversement. Toutefois, le rapprochement effectué entre en bloc et globalement concerne son acception 1 mise en évidence en $\S 2.3$., tandis que celle entre dans l'ensemble et globalement concerne son acception 3. Globalement et en bloc, atteignent ici la totalité, l'intégralité, celle-ci allant à l'encontre du sémantisme approximatif et «non-intégral » de dans l'ensemble. Cette différence sémantique majeure nous semble être un argument assez fort pour éviter le rapprochement fait entre dans l'ensemble et en bloc, et pour plaider pour une distinction entre les différents emplois de globalement développés ci-dessus.

\section{Bilan conclusif}

Au terme de cette analyse, nous pouvons entrevoir un noyau notionnel commun à tous les marqueurs étudiés. Pour chacun d'eux, on effectue un «calcul » prenant en compte un objet ou un ensemble dans ses différents aspects. Ce qui les différencie, c'est la manière dont on opère ce calcul ou le niveau auquel il se situe. Les diverses façons d'opérer ce calcul vont induire des sens différents, faisant intervenir des traits sémantiques spécifiques qui s'apparentent tantôt à la généralité, à l'approximation ou à la quantification totale. La comparaison de dans l'ensemble avec des marqueurs qui lui sont sémantiquement apparentés nous a permis de mettre au jour la complexité sémantique de ce marqueur, à travers les liens qu'il établit. Il présente en effet un ensemble de traits qui permettent d'établir son réseau sémantique, mais aussi de faire ressortir les liens existant entre les autres adverbiaux. On retiendra ainsi pour dans l'ensemble les caractéristiques suivantes :

- un processus d'évaluation qui se fait par approximation : globalement, en gros / grosso modo, en général (opposés à au total qui effectue une sommation, une addition pure dans laquelle il y a considération individuelle des unités);

- la prise en compte d'un ensemble qui laisse place à quelques exceptions : en général, globalement, en gros / grosso modo ;

- un caractère conclusif : au total, globalement, en gros ;

- une modalisation par l'atténuation : globalement.

Globalement apparaît dès lors comme le marqueur le plus proche de dans l'ensemble, puisqu'ils partagent les quatre traits majeurs mis en évidence. Al'inverse, en bloc n'en partage aucun, ce qui plaide pour un non rapprochement de ces deux locutions. Cet exemple nous permet de répondre en partie à l'interrogation de Cruse (2000: 156) concernant le poids des différences possibles entre «synonymes » sur l'intuition d'identité. Si ce n'est l'idée d'ensemble - comme nous l'avons vu ci-dessus - en bloc et dans l'ensemble présentent deux traits opposés, à savoir la complétude et la non complétude, et cette différence doit être considérée comme majeure dans l'établissement des ponts lexicaux. 


\section{Bibliographie}

Anscombre, J.-C. (1990) : «Les syllogismes en langue naturelle. Déduction logique ou inférence discursive ? ", Cahiers de linguistique française, 11, 215240.

Blumenthal, P. (2002) : «Profil combinatoire des noms. Synonymie distinctive et analyse contrastive », Zeitschrift für Französische Sprache und Literatur, $112,115-138$.

Charolles, M. (1984) : "En réalité et en fin de compte et la résolution des oppositions ", Travaux du centre de recherches sémiologiques 47, 81-111.

CRUSE, D.A. (2000) : Meaning in Language. An introduction to semantics and pragmatics, Oxford, Oxford University Press.

EDMONDS, P. \& HiRst, G. (2002) : « Near-Synonymy and Lexical Choice », Computational Linguistics, 28/1, 105-144.

FUCHS, C. (2007) : «Relations de synonymie entre polysèmes : le réseau commemanière-façon », Le Français Moderne, 75 : 1, 97-113.

GeErarts, D. (1994) : « Synonymy», in Asher, R.E. \& Simpson, J.M. (eds), The Encyclopedia of Language and Linguistic, Oxford, New York, Seoul u.a., Pergamon Press, 4455-4456.

GIRARD, G. (1718) : La justesse de la langue françoise, ou les différentes significations des mots qui passent pour synonimes, Paris, L. d'Houry.

GUIMIER, C. (1996) : Les Adverbes du français. Le cas des adverbes en-ment, Paris, Ophrys.

HAßLER, G. (2005) : «Synonymie et incompatibilité des noms d'émotions », Lidil, $32,49-66$.

HuYGHE, R. (à par.) : Les Noms généraux d'espace en français. Enquête linguistique sur la notion de lieu.

JAYEZ, J. \& TOVENA, L. (2008) : «Presque and almost: how argumentation derives from comparative meaning », in Bonami, O.\& Cabredo Hofherr, P. (éds), Empirical issues in syntax and semantics, 7, 217-239, disponible sur http ://www.cssp.cnrs.fr/eiss7

KLEIBER, G. (1983) : « Remarques sur la généricité et la spécificité », Le Français Moderne, 51/1, 36-49.

- (1987): Du côté de la référence verbale. Les phrases habituelles, Berne, Peter Lang.

- (1988) : «Phrases génériques et raisonnement par défaut », Le Français Moderne, 56, 1/2, 1-15.

LAMMERT, M. et VASSILIADOU, H. (à par.) : «Modalités de quantification : le cas de dans l'ensemble », in Kleiber, G. et Schnedecker, C. (éds), La Quantification et ses domaines.

LE FRANÇAIS MODERNE 75 : 1 (2007) : La synonymie en questions. Echanges entre les époques.

LEHMANn, A. \& MARTIN-BERTHET, F. (1998) : Introduction à la lexicologie. Sémantique et morphologie, Paris, Dunod.

LEWIS, D. (1975) : «Adverbs of quantification », in Keenan, E. (ed.), Formal Semantics of Natural Language, Cambridge, Cambridge University Press, 3-15.

LYONS, J. (1970) : Linguistique générale, Paris, Larousse.

MÉLIS, L. (2003) : «Les quantificateurs approximatifs de type prépositionnel », Verbum, XXV, 1, 5-24. 
MOLINIER, C. (2005) : «Les formes adverbiales du français construites sur l'adjectif général », in Goes, J. (éd.), L'Adverbe : un pervers polymorphe, Artois, Artois Presses Université, 89-106.

Molinier, C. et LeVrier, F. (2000) : Grammaire des adverbes, Genève, Droz.

NøJGAARD, M. (1993): Les Adverbes français. Essai de description fonctionnelle, vol. 2, Historisk-filosofiske Meddelelser, 66, Copenhague, Munksgaard.

RossARI, C. (1993) : «A propos de l'influence de la composition morphologique d'une locution sur son fonctionnement sémantico-pragmatique », Cahiers de Linguistique française, 14, 151-171.

- (1994): Les opérations de reformulation: analyse du processus et des marques dans une perspective contrastive français-italien, Berne, Peter Lang.

— (1996) : «Considérations sur la méthodologie contrastive français-italien à propos de locutions adverbiales fonctionnant comme connecteurs », in Hansen, M. B. et Skytte, G. (éds), Le Discours : Cohérence et Connexion, Etudes romanes, 35, 55-68.

SCHNEDECKER, C. (1992) : «La gestion des marqueurs récapitulatifs : l'exemple de bref ", in Charolles, M. et Petitjean, A. (éds), L'Activité résumante, Metz, Centre d'Analyse Syntaxique de l'Université de Metz, 63-104.

- (2008) : « De au total à total : ou comment un quantificateur adverbial en arrive à reformuler et à conclure », in Le Bot, M.-C., Schuwer, M et Richard, E. (éds), La Reformulation : marqueurs linguistiques, stratégies énonciatives, Rennes, PU de Rennes, 127-143.

STEUCKARDT, A. (2005) : «En somme (notice) », in Steuckardt, A. et Niklas-Salminen, A. (éds), Les Marqueurs de glose, Aix-en-Provence, Publications de 1'Université de Provence.

VASSILIADOU, H. (2008) : «Quand les voies de la reformulation se croisent pour mieux se séparer : à savoir, autrement dit, c'est-à-dire, en d'autres termes ", in Le Bot, M.-C., Schuwer, M et Richard, E. (éds), La Reformulation : marqueurs linguistiques, stratégies énonciatives, Rennes, PU de Rennes, 35-50.

VASSILIADOU, H. et LAMMERT, M. (2008) : «Dans l'ensemble : marqueur d'atténuation », in Durand, J., Habert, B. \& Laks, B. (éds), Actes du $1^{\text {er }}$ Congrès mondial de linguistique française, disponible sur http ://dx.doi.org/10.1051/cmlf08107 